\title{
Estimation of Continous Heel and Toe Clearances Using Foot-Worn Inertial Sensors
}

\author{
Yi Zhang ${ }^{1}$, Yinfeng $\mathrm{Wu}^{1, *}$, Jing $\mathrm{Nie}^{1}$, Yang $\mathrm{Yu}^{2}$ \\ ${ }^{1}$ School of Instrumentation and Optoelectronic Engineering, Beihang University, Beijing, 100191China \\ ${ }^{2}$ Faculty of Engineering and Information Technology, \\ University of Technology Sydney, Sydney, NSW 2007, Australia \\ *Corresponding author, Email: yfwu@buaa.edu.cn
}

\begin{abstract}
Falls happen frequently to older people in their daily life. Heel and toe clearances are regarded as significant indicators for the risk of falling. In this study, we developed a foot-worn inertial system to capture kinematic gait data. An estimation method of continuous heel and toe clearances during walking was proposed. Based on heel strike and toe off events detection, a foot movement model was presented to calculate the continuous heel and toe clearances. To compensate the sensor drift, an algorithm combined Zero Velocity Update, Zero Angular Rate Update, Zero Vertical Displacement Update plus a liner drift correction model was implemented. To verify the effectiveness of the drift compensation algorithm, the calculated IMU clearances before and after the implementation of the algorithm were compared and the walking tests results supported its feasibility. Furthermore, the continuous heel and toe clearances were estimated successfully using the foot movement model.
\end{abstract}

Keywords-inertial sensors; foot-worn; foot clearance; foot movement model

\section{INTRODUCTION}

Gait abnormalities are particularly common in the elderly, among which the occurrence of falls happens most frequently in their daily life. One of the major causes of falling is tripping, which could happen directly with deficient or unstable foot clearance. Thus, foot clearance, the vertical distance between the foot and the ground, has been recognized as a significant gait parameter related to the risk of falling [1].

Many researches have used different measuring instruments to obtain foot clearance. The most accurate measuring system is optical motion capture system, but it is costly and can only be used within the laboratory. Andy Kerr et al. used an optical proximity sensor attached to the shoe to measure foot clearance during gait [2]. Yongbin Qi et al. used wearable wireless ultrasonic sensor network to measure 3-dimentional foot displacement [3]. However, their method has the problem of limited application scope and subjects can only implement the experiments on the treadmill. Due to the advantages of being low-cost, wearable and portable, inertial sensors are also selected as the measuring instruments to obtain foot clearance.
Many previous studies focused on the estimation of minimum clearance. Daniel T.H. Lai et al. placed the sensing device at the distal end of the shoe to estimate toe clearance [4]. In [5], the inertial sensor was attached to distal end of the right foot to estimate minimum toe clearance. Naoki Kitagawa et al. attached an inertial measurement unit to the dorsum of the foot, regarding the vertical trajectory of sensor as the foot clearance [6]. Mariani et al. [1] are the few ones to research the estimation of both heel and toe clearances during walking. However, the calculation model is complex, which relied on the sensor location information with respect to the heel and toe. Further investigation for the heel and toe clearances with more simplified model during walking is valuable for application of gait assessment during daily life.

The purpose of this paper was to establish a method for the estimation of continuous heel and toe clearances during walking. In this study, a light-weight, foot-worn gait monitoring system was developed using inertial sensors. We investigated the partitioning of gait cycle and the detection of gait events. Kinematic inertial signals were analyzed and the optimal features for heel strike and toe off events were determined under the reference of pressure signals. The strapdown inertial integration algorithm was introduced to obtain the trajectory of sensor. Then a foot movement model was constructed to calculate the continuous heel and toe clearances. Furthermore, we verified the effectiveness of the drift compensation algorithm, which combined several approaches.

\section{METHODS}

\section{A. Measurenment devices and sensor installation}

A 9-axis inertial measurement unit (IMU) MPU-9250 was used to collect gait data, which integrated a 3-axis gyroscope (range $\pm 2000^{\circ} / s$ ), 3-axis accelerometer (range $\pm 16 g$ ), 3axis magnetometer (range $\pm 4800 \mu T$ ) and a Digital Motion Processor. The inertial sensor unit was embedded in the insole, with three membrane pressure sensors glued to the bottom of the insole (see Fig.1(a)), devised as a reference system for gait 


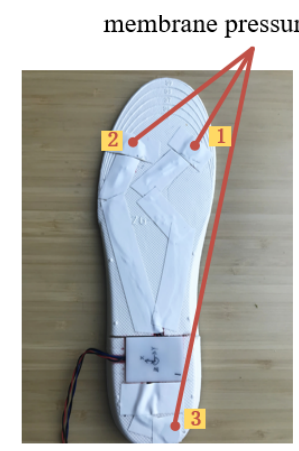

(a)

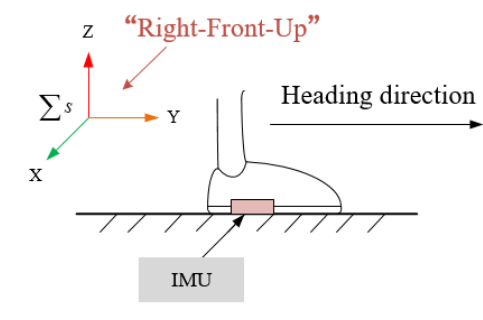

(b)
Figure 1. (a) The installation of IMU. Two membrane pressure sensors were placed in the toe area, with one placed in the heel area, as the reference system for gait events detection. (b) Schematic diagram of sensor axes.

events detection. The sensor axes are shown in Fig.1(b), referred as 'Right-Front-Up' coordinate system.

\section{B. Gait events detection}

Depending on different clinical application requirements, gait cycle was divided into different partitioning models [9]. In our research, we divided gait cycle into four phases, i.e. loading response, foot flat, push and swing phase (see Fig.2.). During normal walking, the foot will experience the following key gait events in each complete gait cycle, i.e. Heel Strike (HS), Toe Strike (TS), Heel Off (HO) and Toe Off (TO). Additionally, stance phase is defined as the period between HS and TO, during which the foot is in contact with the ground.

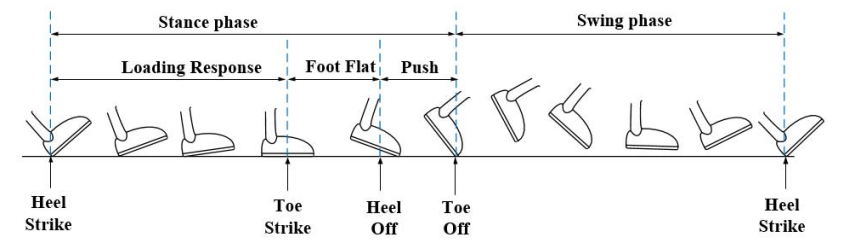

Figure 2 Gait cycle partitioning and gait events

1) Analysis of kinematic inertial signals and pressure signals

Gait events detection depends on the extraction of feature points on the kinematic inertial signals. K.Aminian et al. demonstrated that HS and TO occurred at negative peaks of the foot pitch angular velocity signal [10]. Benoit Mariani et al. investigated three inertial signals, including foot pitch angular velocity, the norm of $3 \mathrm{D}$ accelerometer signal and the derivative of 3D gyroscope signal and extracted the minimum, maximum and zero-crossing feature points respectively [11]. The results showed that the norm of $3 \mathrm{D}$ accelerometer signal could more accurately detect HS and TO, while HS appeared at a trough and TO appeared at a peak in the $3 \mathrm{D}$ accelerometer signal.

In our study, the most commonly used foot pitch angular velocity signal $\left(\omega_{p}\right)$ and the norm of $3 \mathrm{D}$ accelerometer signal $(\|\boldsymbol{a}\|)$ were selected as the characteristic signals for gait events detection. The pressure signals were used as reference signals. Due to individual differences in walking, there were differences in the toe areas where the foot first landed and left the ground. In order to ensure that the pressure of the toe areas could be detected, two membrane pressure sensors were installed. And the abrupt characteristic points of the pressure signals were used as reference points for gait events.

\section{2) Detection of $H S$ and $T O$}

When foot hits the ground, there is a buffering process, and heel strike event actually continues for a very short period. Some studies identified HS at the end of the buffering process, typically appearing at the negative peak of $\omega_{p}[10][12]$. While in this study, HS was detected immediately after the foot hits the ground. Through preliminary signals analysis, the first positive peak $P_{1}$ of $\|\boldsymbol{a}\|$ and the zero-crossing sampling point $P_{2}$ of $\omega_{p}$ were considered as candidate features for detecting HS. As for TO, the second positive peak $P_{3}$ of $\|\boldsymbol{a}\|$ and the negative peak $P_{4}$ of $\omega_{p}$ were the candidate features, as seen in Fig.3.
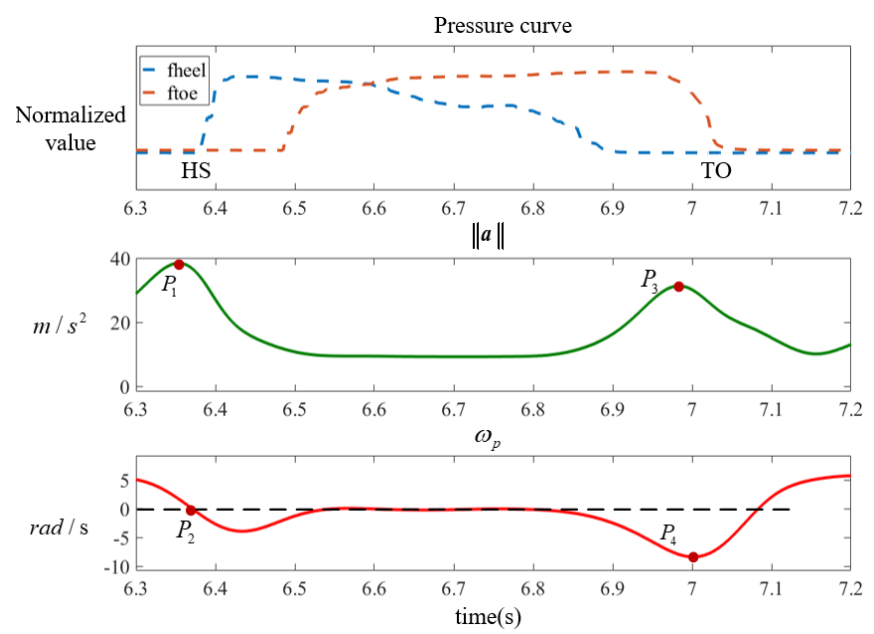

Figure 3 Candidate features for detecting HS and TO.

To obtain the optimal feature set for detecting HS and TO, we implemented a simple experiment. Two healthy individuals (one male, one female) without discernable gait abnormalities were recruited to perform our walking test, wearing the right size insoles embedded with foot-worn inertial sensors and pressure reference system. Each subject walked on the flat corridor at the normal pace of his own habit for about $24 \mathrm{~m}$, and each subject repeated three times of experiments.

A total of 113 gait cycles were detected, including $113 \mathrm{HS}$ and 113 TO. The differences between the candidate features and the reference points were calculated respectively. Table I summarized the mean and standard deviation of absolute errors of HS and TO. The results showed that the optimal feature set for detecting $\{\mathrm{HS}, \mathrm{TO}\}$ was $\left\{P_{2}, P_{4}\right\}$.

\section{3) Detection of foot flat}

Foot flat phase corresponds to the period between TS and $\mathrm{HO}$, during which the whole foot remains stationary with the ground. Ideally, the angular rate, the velocity, and the 
translational acceleration of the foot equal to zero in this stage. A Generalized Likelihood Rate Tests Detector [13] was used to detect foot flat phase, which integrated acceleration and angular velocity data, avoiding the limitation of using only one of them as the test standard.

TABLE I. MEAN AND STANDARD DEVIATION OF ABOSOLUTE DIFFERENCES BETWEEN CANDIDATE FEATURES AND REFERENCE POINTS

\begin{tabular}{|c|c|c|c|}
\hline \multirow{2}{*}{ Gait Events } & $\begin{array}{c}\text { Candidate } \\
\text { features }\end{array}$ & Mean(s) & STD(s) \\
\hline \multirow{2}{*}{ HS } & $P_{1}$ & 0.008 & 0.016 \\
\cline { 2 - 4 } & $P_{2}$ & 0.007 & 0.011 \\
\hline \multirow{2}{*}{ TO } & $P_{3}$ & 0.042 & 0.031 \\
\cline { 2 - 4 } & $P_{4}$ & 0.008 & 0.006 \\
\hline
\end{tabular}

C. Strapdown inertial integration algorithm of the sensor trajectory

\section{1) Sensor orientation update}

In this study, we adopted the rotation quaternion to describe the sensor orientation at sample $k$ during walking, defined as follows:

$$
\boldsymbol{q}(k)=\left[\begin{array}{llll}
q_{0} & q_{1} & q_{2} & q_{3}
\end{array}\right]
$$

$\boldsymbol{q}(k)$ was updated using the gyroscope data and the initial sensor orientation, which was estimated by the acceleration data at initial stationary stage. The rotation quaternion can be updated by the following differential equation:

$$
\dot{\boldsymbol{q}}=\frac{1}{2} \Omega \boldsymbol{q}
$$

where $\Omega=\left[\begin{array}{cccc}0 & -\omega_{x} & -\omega_{y} & -\omega_{z} \\ \omega_{x} & 0 & \omega_{z} & -\omega_{y} \\ \omega_{y} & -\omega_{z} & 0 & \omega_{x} \\ \omega_{z} & \omega_{y} & -\omega_{x} & 0\end{array}\right]$.

\section{2) Velocity and position estimation}

The estimation of velocity and position is based on the integration of foot acceleration data on the navigation frame $\left(\sum n\right)$. Since the measured acceleration data were represented by the sensor frame $\left(\sum s\right)$, it should be transformed to the navigation frame.

The sensor coordinate system ( $\sum s$ ) is aligned with the foot coordinate system $\left(\sum b\right)$ because of the configuration placement, therefore, the two systems could be regarded as the same frame. And we made the navigation frame $\left(\sum n\right)$ equal to the local horizontal rectangular frame. The transformation matrix $\boldsymbol{C}_{b}^{n}$ can be calculated as:

$$
\boldsymbol{C}_{b}^{n}=\left[\begin{array}{ccc}
q_{0}^{2}+q_{1}^{2}+q_{2}^{2}+q_{3}^{2} & 2\left(q_{1} q_{2}-q_{0} q_{3}\right) & 2\left(q_{1} q_{3}+q_{0} q_{2}\right) \\
2\left(q_{1} q_{2}+q_{0} q_{3}\right) & q_{0}^{2}-q_{1}^{2}+q_{2}^{2}-q_{3}^{2} & 2\left(q_{2} q_{3}-q_{0} q_{1}\right) \\
2\left(q_{1} q_{3}-q_{0} q_{2}\right) & 2\left(q_{2} q_{3}+q_{0} q_{1}\right) & q_{0}^{2}-q_{1}^{2}-q_{2}^{2}+q_{3}^{2}
\end{array}\right]
$$

After the transformation, the gravitational acceleration should be removed from the measured acceleration vector $\boldsymbol{a}_{k}^{b}$. Then the useful transitional acceleration vector $\boldsymbol{a}_{k}^{n}$ could be calculated as:

$$
\boldsymbol{a}_{k}^{n}=\boldsymbol{C}_{b}^{n} \boldsymbol{a}_{k}^{b}-[0,0, \mathrm{~g}]^{T}
$$

The velocity $\boldsymbol{v}_{k}$ was obtained by the trapezoidal integration of $\boldsymbol{a}_{k}^{n}$, and the position of IMU $\boldsymbol{r}_{k}$ was estimated by the trapezoidal integration of $\boldsymbol{v}_{k}$ :

$$
\begin{gathered}
\boldsymbol{v}_{k}=\boldsymbol{v}_{k-1}+\left(\boldsymbol{a}_{k}^{n}+\boldsymbol{a}_{k-1}^{n}\right) / 2 \cdot \Delta t \\
\boldsymbol{r}_{k}=\boldsymbol{r}_{k-1}+\left(\boldsymbol{v}_{k}^{n}+\boldsymbol{v}_{k-1}^{n}\right) / 2 \cdot \Delta t
\end{gathered}
$$

where $\Delta t$ represents the sampling interval.

\section{Estimation of continious heel and toe clearances}

In this paper, a foot movement calculation model was constructed to estimate continuous heel and toe clearances during walking. During swing phase, the foot has two tilting states, seen in Fig.4. When the foot is inclined towards the heading direction, the pitch angle $\theta(k)$ is negative. Under two tilting states, the heel $h_{\text {heel }}(k)$ and toe clearance $h_{\text {toe }}(k)$ can be computed based on the vertical position of IMU $h_{i m u}(k)$, also referred as IMU clearance, which is the projection of $\boldsymbol{r}_{k}$ on the z-axis:

$$
\left\{\begin{array}{c}
h_{\text {heel }}(k)=h_{\text {imu }}(k)-d_{\text {heel }} \cdot \sin (\theta(k)) \\
h_{\text {toe }}(k)=h_{\text {imu }}(k)+d_{\text {toe }} \cdot \sin (\theta(k))
\end{array}\right.
$$

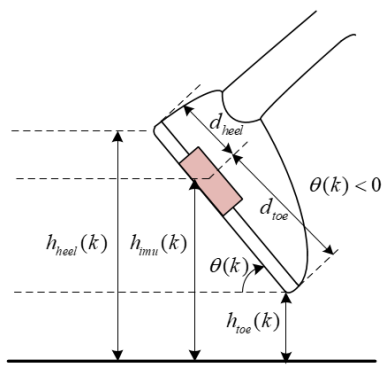

(a)

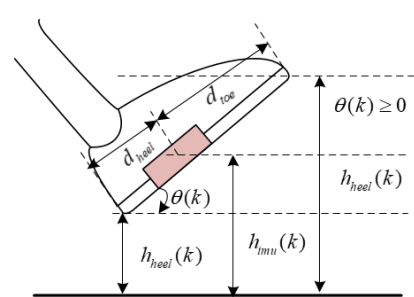

(b)
Figure 4 The foot movement calculation mode

The distances between IMU and heel $d_{\text {heel }}$ or the toe $d_{\text {toe }}$ can be estimated using biological motion hypotheses without the information of shoe sizes and the installation displacement of IMU. Assuming that the foot does not deform during HS and TO, $d_{\text {heel }}$ and $d_{\text {toe }}$ can be estimated during HS and TO respectively:

$$
d_{\text {heel }}=\frac{h_{\text {imu }}(H S)}{\sin (\theta(H S))}, d_{\text {toe }}=-\frac{h_{\text {imu }}(T O)}{\sin (\theta(T O))}
$$

\section{E. Compensation algorithm of sensor drift}

One of the biggest challenges of using inertial sensors is to compensate the integrated sensor drift. If the sensor data were integrated continually without any correction, the velocity error would increase linearly, and the position error would increase 
at least quadratically [14]. In this study, several methods were used. To correct the integration drift, we firstly implemented the Zero-Velocity Update (ZUPT) technology [15] and the Zero-Angular Rate Update (ZARU) [16] technology during foot flat phase. The velocity and the angular rate of the foot were reset to zero during foot flat, avoiding the accumulation of drift error during different gait cycles. In addition, a Zero Vertical Displacement Update (ZVDU) method was proposed in this study. In [6] similar work was mentioned as well. ZVDU relied on the assumption that the ground slope is negligible during walking, thus, the vertical displacement of the foot (foot clearance) during each foot flat phase should be zero, which is also an additional motion constraint than the horizontal displacement. Therefore, IMU clearance, heel and toe clearances were corrected to zero during each foot flat.

Theoretically, if the velocity and the clearances are reset to zero during foot flat, after the integration, they should be zero at the beginning of next foot flat. In practical, the existed sensor error will make the integrated data unequal to zero. Thus, we applied a liner drift correction model (short for LC) [17] to compensate the drift on the velocity and the clearances between the adjacent foot flats.

\section{RESULTS}

\section{A. Verification of the compensation algorithm}

Several healthy individuals without discernable gait abnormalities were recruited to perform the walking test. Each subject walked on the flat corridor at the self-selected speed for around $24 \mathrm{~m}$, wearing the right size insoles embedded with foot-worn inertial sensors. Raw gait data were low-pass filtered at $10 \mathrm{~Hz}$ [7][8], sampled at $200 \mathrm{~Hz}$.

To verify the effectiveness of the compensation algorithm of sensor drift, the calculated continuous IMU clearances during walking before and after the implementation of compensation algorithm were compared. Fig.5 depicted a typical walking test result. The result showed that if we directly used the strapdown inertial integration algorithm to calculate the continuous IMU clearance, the drift would keep accumulated over time. After the drift compensation by combining ZUPT, ZARU, ZVPU and LC, it showed a reasonable characteristic curve of continuous IMU clearance.

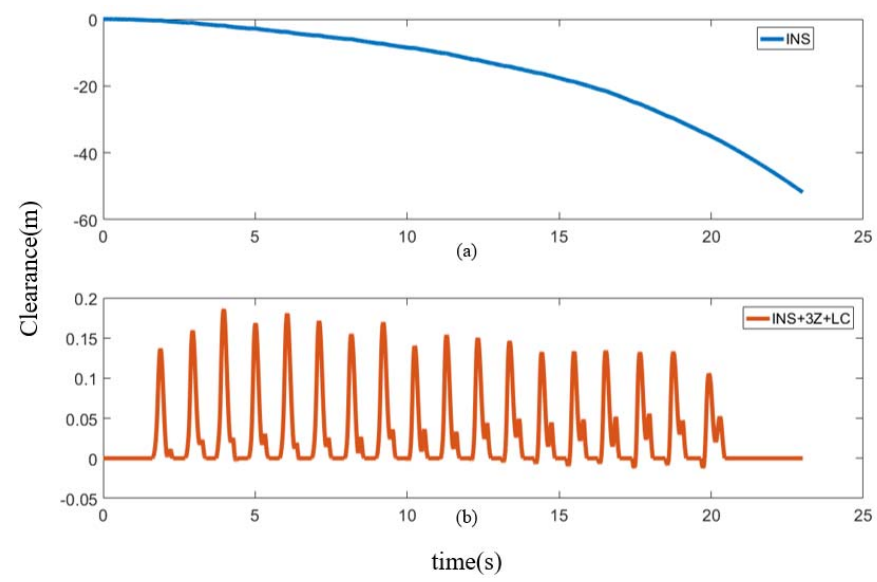

Figure 5 The continuous IMU clearances during walking before and after the drift compensation. a) The calculated IMU clearance using the strapdown integration algorithm (marked as INS). b) The calculated IMU clearance which combined INS and ZUPT, ZARU, ZVPU, LC (marked as INS+3Z+LC).

\section{B. Evaluation of the proposed method}

To evaluate the feasibility of this study, we compared the proposed method with previous method mentioned in[6], in which the authors also used a wearable inertial measurement unit mounted to the foot to estimate the foot clearance. The difference is that they only measured the clearance of IMU. To unify the comparison standard, we only compared the IMU clearances estimated by our method and theirs.

Fig.6 showed a good correspondence between two IMU clearances calculated by the proposed method and the method mentioned in [6] during a typical recorded gait cycle. During the swing phase, IMU clearance will reach a maximum value (MaxFC1) after toe off. Then before heel strike, IMU will reach a minimum value (MinFC) and a second maximum value (MaxFC2).

Table II showed the mean absolute difference values of the three parameters calculated by the two methods during five walking tests. The results showed the proposed method could produce comparable IMU clearances compared with the method in [6].

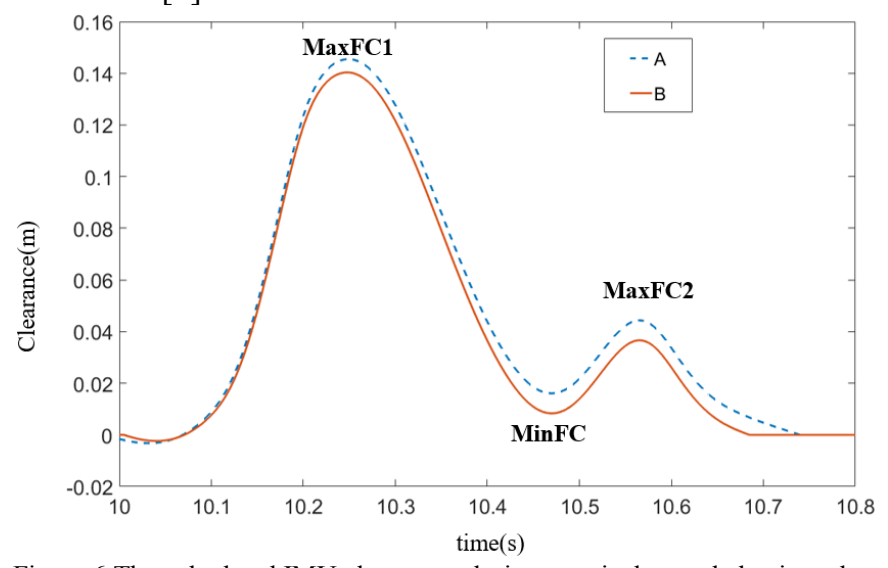

Figure 6 The calculated IMU clearances during a typical recorded gait cycle using the proposed method (marked as B) and the method mentioned in [6] (marked as A).

TABLE II. THE MEAN ABSOLUTE DIFFERENCES OF MAXFC1, MAXFC2, MINFC BETWEEN THE TWO REUSLTS CALCULATED BY THE TWO METHODS

\begin{tabular}{|c|c|c|c|c|}
\hline Test & Gait Cycle & MaxFC1 (mm) & MaxFC1 (mm) & MinFC (mm) \\
\hline 1 & 18 & 2.3 & 4.0 & 3.9 \\
\hline 2 & 21 & 2.1 & 2.4 & 2.5 \\
\hline 3 & 18 & 4.1 & 1.8 & 3.5 \\
\hline 4 & 18 & 2.6 & 3.6 & 3.3 \\
\hline 5 & 19 & 2.7 & 2.6 & 2.7 \\
\hline
\end{tabular}

\section{Results of continous heel and toe clearances}

After calculation and correction of the continuous IMU clearance, the continuous heel and toe clearances during walking were estimated based on the foot movement calculation model, both of which were calculated at each sample point. Fig. 7 showed the typical continuous heel and toe clearance characteristic signals among walking tests. 


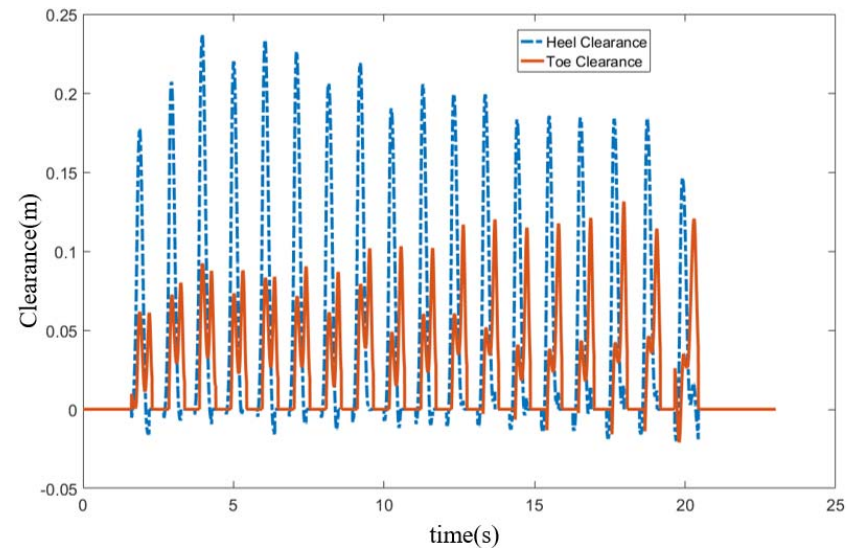

Figure 7 The continuous heel and toe clearances during one walking test.

\section{DISCUSSION AND CONCLUSION}

According to the results, the calculated heel and toe clearances exhibited periodic characteristics in each gait cycle, which corresponded to the previous studies. The heel clearance has one maximum (MaxHC) after toe off, and the toe clearance has two maximums (MaxTC1, MaxTC2) and one minimum (MinTC), as shown in Fig.8. The further experiment is needed to study these clearance parameters to better understand human gait.

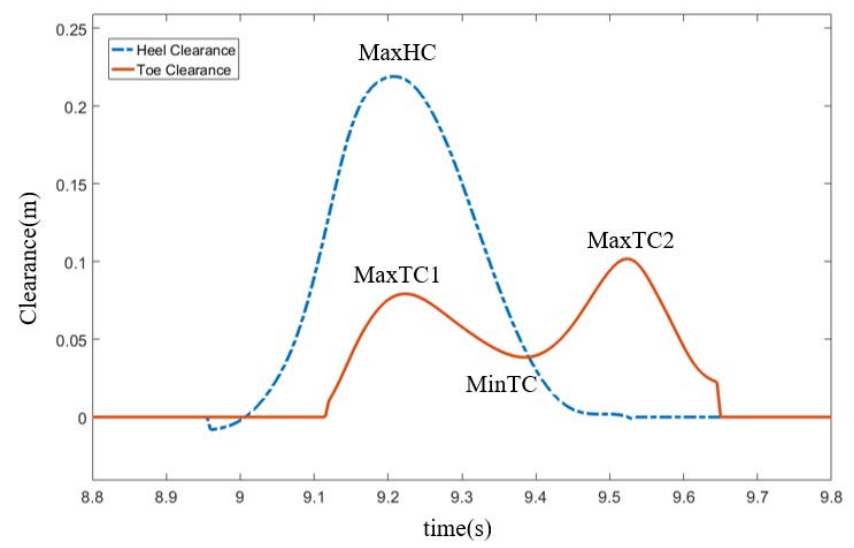

Figure 8 The clearance parameters in each gait cycle

In summary, this study established a method for estimation of continuous heel and toe clearances using a foot-worn inertial system. Gait cycle was divided into four phases, and three pressure sensors were used as the reference system for gait events detection. The experiment results showed that the optimal features for detecting $\mathrm{HS}$ and TO were $P_{2}$ and $P_{4}$ respectively. The constructed movement calculation model proved to be feasible to estimate the continuous heel and toe clearances. Furthermore, the compensation algorithm we implemented effectively corrected the integration drift. This foot-worn inertial system we developed may serve as a useful tool for gait assessment of subjects without gait disorder in their daily life.

\section{ACKNOWLEDGMENT}

We acknowledge all the participants who contributed to this study. This study is supported in part by the Defense Industrial Technology Development Program (Grant: JSJL2017601B001).

\section{REFERENCES}

[1] B. Mariani, S. Rochat, J. B. Christophe, and K. Aminian, "Heel and Toe Clearance Estimation for Gait Analysis Using Wireless Inertial Sensors," vol. 59, no. 11, pp. 3162-3168, 2012.

[2] A. Kerr, "Using an Optical Proximity Sensor to Measure Foot Clearance During Gait :," vol. 4, no. September 2010, pp. 1-5, 2018.

[3] Y. Qi, C. B. Soh, E. Gunawan, and K. Low, “Ambulatory Measurement of 3-Dimensional Foot," vol. 2194, no. c, pp. 1-8, 2014.

[4] D. T. H. Lai, E. Charry, R. Begg, S. I. Member, M. Palaniswami, and S. I. Member, "A prototype wireless inertial-sensing device for measuring toe clearance," pp. 4899-4902, 2008.

[5] B. K. Santhiranayagam, D. T. H. Lai, W. A. Sparrow, and R. K. Begg, "A machine learning approach to estimate Minimum Toe Clearance using Inertial Measurement Units," J. Biomech., vol. 48, no. 16, pp. 4309-4316, 2015.

[6] N. Kitagawa and N. Ogihara, "Estimation of foot trajectory during human walking by a wearable inertial measurement unit mounted to the foot," vol. 45, pp. 110-114, 2016.

[7] A. G. Byju, M. A. Nussbaum, and M. L. Madigan, "Alternative measures of toe trajectory more accurately predict the probability of tripping than minimum toe clearance," vol. 49, pp. 4016-4021, 2016.

[8] D. Mcgrath, B. R. Greene, C. Walsh, and B. Caulfield, "Estimation of minimum ground clearance ( MGC ) using body-worn inertial sensors," J. Biomech., pp. 1-6, 2011.

[9] J. Taborri, E. Palermo, S. Rossi, and P. Cappa, "Gait partitioning methods: A systematic review," Sensors (Switzerland), vol. 16, no. 1, pp. 40-42, 2016.

[10] P. Leyvraz and P. Robert, "Spatio-temporal parameters of gait measured by an ambulatory system using miniature gyroscopes," vol. 35, pp. 689699, 2002.

[11] B. Mariani, H. Rouhani, X. Crevoisier, and K. Aminian, "Quantitative estimation of foot-flat and stance phase of gait using foot-worn inertial sensors," Gait Posture, vol. 37, no. 2, pp. 229-234, 2013.

[12] B. R. Greene, D. McGrath, R. O’Neill, K. J. O’Donovan, A. Burns, and B. Caulfield, "An adaptive gyroscope-based algorithm for temporal gait analysis," Med. Biol. Eng. Comput., vol. 48, no. 12, pp. 1251-1260, 2010.

[13] I. Skog, H. Peter, S. Member, J. Nilsson, and J. Rantakokko, "ZeroVelocity Detection - An Algorithm Evaluation," vol. 57, no. 11, pp. 2657-2666, 2010.

[14] C. Fischer, "Implementing a Pedestrian Tracker Using Inertial Sensors," pp. 17-27, 2013.

[15] Z. Wang, H. Zhao, S. Qiu, and Q. Gao, "Stance phase detection for ZUPT-aided foot-mounted pedestrian navigation system," vol. 4435, no. c, pp. 1-12, 2015.

[16] A. R. Jim, F. Seco, J. C. Prieto, and J. Guevara, "Indoor Pedestrian Navigation using an INS / EKF framework for Yaw Drift Reduction and a Foot-mounted IMU," pp. 135-143, 2010.

[17] S. Yang, C. Mohr, and Q. Li, "Ambulatory running speed estimation using an inertial sensor," Gait Posture, vol. 34, no. 4, pp. 462-466, 2011 